Article

\title{
Comprehensive Evaluation of AC/DC Hybrid Microgrid Planning Based on Analytic Hierarchy Process and Entropy Weight Method
}

\author{
Guopeng Zhao *(D) and Dong Wang \\ School of electrical and electronic engineering, North China Electric Power University, Beijing 102206, China; \\ wd33666@163.com \\ * Correspondence: zhaoguopeng@ncepu.edu.cn; Tel.: +86-010-61771713
}

Received: 31 July 2019; Accepted: 10 September 2019; Published: 12 September 2019

check for updates

Featured Application: Authors are encouraged to provide a concise description of the specific application or a potential application of the work. This section is not mandatory.

\begin{abstract}
The comprehensive evaluation of AC/DC hybrid microgrid planning can provide reference for the planning of AC/DC hybrid microgrids. This is conducive to the realization of reasonable and effective microgrid planning. Aiming at comprehensive evaluation of AC/DC hybrid microgrids, this paper establishes an evaluation index system for planning of AC/DC hybrid microgrids. This paper combines the subjective evaluation method with the objective evaluation method, and proposes a comprehensive evaluation method of AC/DC hybrid microgrid planning based on analytic hierarchy process and the entropy weight method. Finally, the validity and rationality of the evaluation method are verified by an example.
\end{abstract}

Keywords: analytic hierarchy process; entropy weight method; grey relevance degree; comprehensive evaluation; microgrid

\section{Introduction}

At present, in order to solve the problem of environmental protection and energy exhaustion, renewable clean energy has a high proportion of access. This also brings many new challenges, such as the low energy density of new energy, the need for suitable sites for high-power power generation, power instability, and so on. The microgrid technology provides an important technical direction for the efficient use of these new energy sources [1-4]. However, in the evaluation of microgrid planning, besides satisfying certain reliability of power supply, it also requires comprehensive consideration of investment costs, power quality, and environmental protection and other factors. Therefore, for such a planning problem with a large number of uncertainties, it is necessary to establish a comprehensive technical and economic evaluation system.

In the aspect of comprehensive evaluation methods, traditional comprehensive evaluation methods mostly use subjective expert evaluation decision-making methods based on analytic hierarchy process (AHP) and fuzzy comprehensive evaluation method. In [5] the authors present a risk-based vulnerability assessment method for microgrids. It uses AHP and the fuzzy comprehensive evaluation method to calculate the comprehensive vulnerability of microgrids. This method relies too much on the subjective judgment of experts to determine the evaluation weight and lacks sufficient objectivity. In [6] the authors use a multi-operator model to construct a hierarchical fuzzy evaluation model. Using the idea of analytic hierarchy process and fuzzy evaluation, the weight of the index system is analyzed, and the calculation method of the index is obtained. This method still has the problem of 
overweight subjectivity. The multi-operator model cannot fundamentally solve the shortcoming of the lack of objectivity of the fuzzy evaluation method. In [7] the authors propose a comprehensive evaluation index system of an AC and DC microgrid based on the Improved Analytic Hierarchy process. However, the improved analytic hierarchy process (AHP) only improves the method of judgment matrix generation. In essence, it still adopts the subjective method of AHP, which has the disadvantage of strong subjectivity of evaluation index weight. In [8] the authors use the analytic hierarchy process and fuzzy comprehensive evaluation method to determine the weight of the evaluation index. In [9] the authors construct the fuzzy evaluation vector of power grid risk by the method of fuzzy comprehensive evaluation. Then, the grid risk level is output according to the principle of maximum membership degree. In [10] the authors establish a fuzzy comprehensive evaluation model for the upgrade demand of smart grid functional form. Then, it uses the fuzzy comprehensive evaluation method to determine the index weight. In [11] the authors establish the evaluation model of power grid equipment utilization rate by using an analytic hierarchy process. In [12] the authors establish a comprehensive evaluation index system for power grid science and technology projects by using the principle of analytic hierarchy process. All the methods used in the above literature are subjective evaluation methods. Although there are some improvements in the process of implementation, it is still unable to overcome the problem of insufficient objectivity of subjective methods in determining the weight of indicators.

In order to solve the problem of insufficient objectivity of traditional methods, objective empowerment methods have been introduced into the comprehensive evaluation of planning schemes in recent years. In [13] the authors combine information entropy theory with principal component analysis theory and apply this method to comprehensive evaluation of thermal power units. It establishes a comprehensive evaluation model of unit state based on information entropy and principal component analysis. However, the principal component analysis method has difficultly solving the problem of high-order correlation between indicators when extracting principal components, which will lead to errors. In [14] the authors use the method of entropy weight to analyze and evaluate the operation state characteristics of power grid. In [15] the authors use the method of entropy weight to determine the weight of distribution project evaluation. In [16] the authors introduce the method of entropy weight to determine the weight coefficient of gas index in oil. The above literature uses objective methods such as entropy weight method as the evaluation model. The advantage of the objective evaluation method is that it can make full use of the original data in the evaluation process. However, its disadvantage is that there will be some errors when the original data is inadequate or inaccurate. In [17] the authors present a method for evaluating the main grid structure based on the improved approximation ideal method and grey relational projection method. It combines subjective and objective evaluation methods to determine weight. However, the subjective evaluation method G1 used in [17] will reduce the differences between indicators when determining the weight of indicators [18]. The result is inaccurate because of errors in determining weights.

A comprehensive evaluation method of AC/DC hybrid microgrid planning based on the analytic hierarchy process and entropy weight method is proposed in this paper. Firstly, this paper establishes a scientific and comprehensive evaluation index system for AC/DC hybrid microgrid planning. Then, the subjective and objective weights are determined based on the AHP and the entropy weight method. According to the principle of minimum information discrimination, the combined weights of subjective and objective weights are obtained. In this paper, a comprehensive evaluation model of AC/DC hybrid microgrid planning is established based on combination weight and grey correlation analysis. Finally, four kinds of microgrid schemes are analyzed by using the comprehensive evaluation method in this paper. Through the method described in this paper, the ranking and optimal scheme of each scheme are obtained. The result is in accordance with the reality. The comprehensive evaluation method described in this paper combines the advantages of the subjective evaluation method and objective evaluation method. It can obtain a more accurate index weight value. This method can not only enhance the objectivity of index weight, but also reduce the errors caused by insufficient or missing 
data, making the evaluation results more scientific and in line with the actual situation. Therefore, the comprehensive method described in this paper has a certain practical value in engineering.

\section{Comprehensive Evaluation Method for AC/DC Hybrid Power Network Planning}

\subsection{Evaluation Index System for AC/DC Hybrid Microgrid Planning}

Constructing an index system is an important task of comprehensive evaluation. Whether the index system is perfect or not directly reflects the quality of comprehensive evaluation. At this stage, there are two mature principles to describe. First, the index system should be comprehensive and not overlapping, and the index can be easily obtained. Secondly, the index system should be able to objectively, scientifically, and reasonably reflect the problems to be studied.

Based on the principles of comprehensive, objective, and typical evaluation index construction, this paper divides the evaluation index system of AC/DC hybrid microgrid planning into four parts: economy, power quality, reliability, and environmental protection. The comprehensive evaluation index system of AC/DC hybrid microgrid is shown in Figure 1.

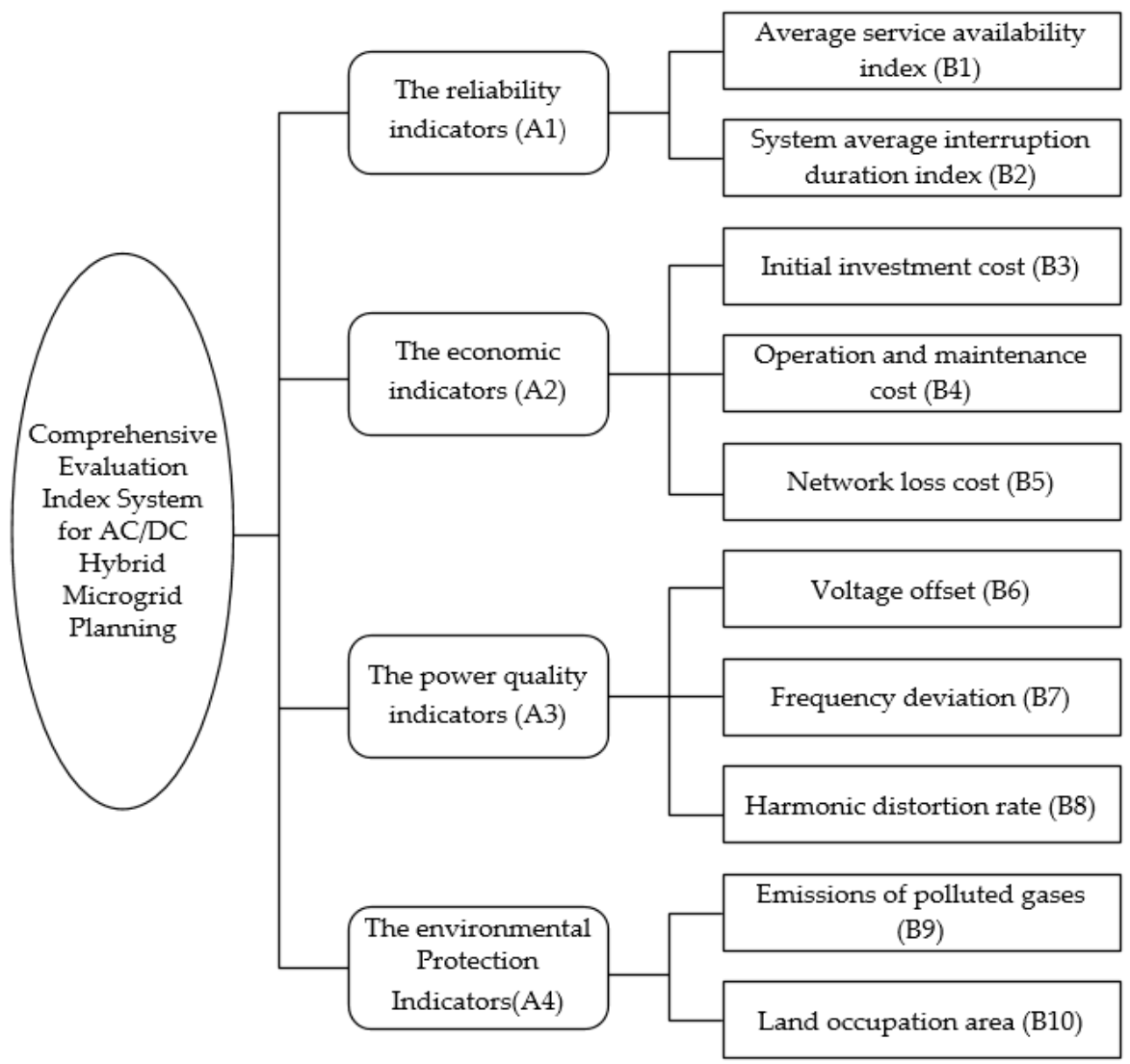

Figure 1. Comprehensive evaluation index system for AC/DC hybrid microgrid planning.

\subsubsection{The Reliability Indicators}

The reliability indicators are mainly divided into two parts, the average service availability index and system average interruption duration index.

Average service availability index (ASAI) [19].

$$
A S A I=1-\frac{\sum U_{i} N_{i}}{8760 \sum N_{i}}
$$


System average interruption duration index (SAIDI)

$$
S A I D I=\frac{\sum U_{i} N_{i}}{\sum N_{i}}
$$

In Equations (1) and (2), $U_{i}$ represents the annual average interruption duration at the load point. $N_{i}$ represents the number of users at the load point.

\subsubsection{The Economic Indicators}

The economic indicators include three parts: initial investment cost, operation and maintenance cost, and network loss cost [20].

$$
\begin{gathered}
f_{D G 1}=\sum_{t=1}^{T} \frac{1}{(1+r)^{t}} \sum_{t=1}^{N} \omega_{D G i} f_{d g 1} \eta_{D G} S_{D G i}+\sum f_{L i} \cdot L_{i}+\sum f_{T_{i}}, \\
f_{D G 2}=t_{D G \max } \sum_{t=1}^{T} \frac{1}{(1+r)^{t+0.5}} \sum_{i=1}^{N} \omega_{D G i} f_{d g 2} P_{D G i} .
\end{gathered}
$$

In Equations (3) and (4), DG represents Distributed Generation. $f_{D G 1}$ is the initial investment cost. $f_{D G 2}$ is the operation and maintenance cost. $T$ is the planning life. $r$ is the discount rate. $N$ is the total number of load nodes. $\omega_{D G 1}$ indicates whether distributed power is installed at node $i .0$ is not installed and 1 is installed. $f_{d g} 1$ is the initial investment cost per unit capacity of distributed generation. $\eta_{D G}$ is the power factor. $S_{D G i}$ is the installed capacity of distributed power supply. $t_{D G \max }$ is the maximum annual working time of distributed power supply. $f_{L i}$ is the unit length cost of transmission line $i . L_{i}$ is the length of line $i . f_{T i}$ is the cost of transformer or converter $i . f_{d g 2}$ is the maintenance cost of unit capacity of distributed power supply. $P_{D G i}$ is the unit output of distributed power supply at node $i$.

$$
f_{\text {LOSS }}=\sum_{t=1}^{T} \frac{C}{(1+r)^{t+1.5}} \sum_{k=1}^{l} R_{k} \frac{P_{L k}^{2}+Q_{L k}^{2}}{V_{k}^{2}} t_{\max k} .
$$

In Equation (5), $f_{\text {LOSS }}$ is the network loss cost. $C$ is the electricity price. $l$ is the total number of branches. $R_{k}$ is the branch resistance. $P_{L k}$ and $Q_{L k}$ are the active and reactive power transmitted by branch $\mathrm{k} . V_{k}$ is the branch $k$ terminal voltage. $t_{\max k}$ is the maximum annual load loss hours of branch $k$.

\subsubsection{The Power Quality Indicators}

The power quality indicators [21,22] have mainly three aspects: voltage offset, frequency deviation, and harmonic distortion rate.

Voltage offset is the difference between the actual voltage and the rated voltage of the network. Voltage offset is often expressed as a percentage. Voltage loss caused by reactive power load and line loss is the main cause of voltage offset. The calculation of voltage offset at each node of power supply system is shown in Equation (6).

$$
\Delta U=\frac{U_{r e}-U_{N}}{U_{N}} \times 100 \% .
$$

In Equation (6), $\Delta U$ is the voltage offset. $U_{r e}$ is the actual voltage. $U_{N}$ is the rated voltage of the system.

Frequency deviation is the difference between the actual value and the nominal value of the system frequency under the normal operation conditions of the power system. According to the regulation, the allowable value of normal frequency deviation of power system is $\pm 0.2 \mathrm{~Hz}$. When the system capacity is small, the deviation can be relaxed to $\pm 0.5 \mathrm{~Hz}$.

$$
\Delta f=f_{1}-50
$$


In Equation (7), $f_{1}$ is the current frequency of the system and $\Delta f$ is the frequency offset of the system. Ideally, the voltage waveform should be a periodic standard sine wave. However, there is much power supply equipment with non-linear impedance characteristics in power systems. These devices inject harmonic current into the public power grid or generate harmonic voltage in the public power grid, which is called harmonic source. Harmonic source makes the actual voltage waveform deviate from sinusoidal waveform, which is called voltage sinusoidal waveform distortion. Usually it is represented by harmonics. The degree of voltage waveform distortion is measured by voltage sinusoidal distortion rate, which is also called voltage harmonic distortion rate. $T_{H D U}$ is commonly used to express the voltage harmonic distortion rate. The total distortion rate of voltage harmonics is calculated as shown in the equation.

$$
T_{H D U}=\frac{\sqrt{\sum_{h=2}^{\infty}\left(U_{h}\right)^{2}}}{U_{1}} \times 100 \% .
$$

In Equation (8), $U_{h}$ is the $h$-th harmonic voltage and $U_{1}$ is the fundamental voltage.

\subsubsection{The Environmental Protection Indicators}

Compared with traditional thermal power generation, DG emits a small amount of polluted gas. It is environmentally friendly. $E$ is defined as the amount of polluted gases emitted by the system [23,24].

$$
\begin{gathered}
e_{k}=\sum_{g=1}^{T_{U}} P_{T R g} e_{T R g k}+\sum_{i=1}^{M} \delta_{a i} P_{f a} S_{D G a} e_{D G a k}, \\
E=8760 \sum_{t=1}^{T} \sum_{k=1}^{B} \omega_{k} e_{k} .
\end{gathered}
$$

In Equations (9) and (10), $E_{K}$ is the $k$ pollutant discharge. $T_{U}$ is the number of traditional generators in the system. $M$ is the total number of load nodes. $T$ is the planned life year. $t$ is the current year. $B$ is the total number of polluted gases, $P_{T R g}$ is the active output of the $g$-th traditional generator, $e_{T R g k}$ is the amount of the $k$ polluted gases released by the $g$-th traditional generator when it emits unit active power. $\delta_{a i}=1$ represents installing DG of type $a$ at the $i$-th load node. While $\delta_{a i}=0$ indicates not to install. $p_{f a}$ is the power factor of $a$-type DG. $S_{D G a}$ is the output of $a$-type DG. $e_{D G a k}$ represents the amount of $k$ polluted gas released by $a$-type DG when it emits unit active power. $A$ is the total number of DG types. $\omega_{k}$ is the weighting factor of the $k$-th pollution gas. $\omega_{k}$ should satisfy Equation (11).

$$
\left\{\begin{array}{c}
\sum_{k=1}^{B} \omega_{k}=1 \\
0 \leq \omega_{k} \leq 1
\end{array}\right.
$$

Table 1 shows the comparison of pollutant emission data between conventional power generation and part of DG [24]. As can be seen from the table, the emissions of pollutants from photovoltaic power generation and wind power generation are zero. Compared with the traditional generation mode, the emission of pollutant gas from distributed generation is lower. 
Table 1. Pollutant emission data of various power generation technologies.

\begin{tabular}{cccc}
\hline Power Generation & \multicolumn{3}{c}{ Pollutant Emissions/(g/kWh) } \\
\cline { 2 - 4 } Mode & $\mathbf{S O}_{\mathbf{2}}$ & $\mathrm{NO}_{\mathbf{X}}$ & $\mathbf{C O}_{\mathbf{2}}$ \\
\hline Thermal power & 6.48 & 2.88 & 623 \\
Micro gas turbine & 0.000928 & 0.6188 & 184.0829 \\
Fuel cell & 0 & $<0.023$ & 635.04 \\
Photovoltaic power & 0 & 0 & 0 \\
Wind power & 0 & 0 & 0 \\
\hline
\end{tabular}

\subsection{Weight Calculation}

At present, there are two main methods to determine the weight: subjective method and objective method. The former mainly includes the analytic hierarchy process, fuzzy evaluation method, expert method, and so on. The latter includes entropy weight method, principal component analysis method, coefficient of variation method, and so on. Among them, the analytic hierarchy process (AHP) is a scientific decision-making method which combines qualitative analysis with quantitative analysis. It has a wide range of applications. However, its shortcoming is that the evaluation process has a part of subjectivity, which reduces the credibility of the evaluation results. Entropy weight method determines the objective weight according to the magnitude of index variability. This method can make better use of the information provided by the original data to explain the results. It has good objectivity. It has been widely used in engineering technology, social economy, and other fields. The disadvantage is that the weight accuracy obtained by the entropy weight method is closely related to the original data, which requires a large amount of data and requires a high accuracy of the data. This paper combines the method of subjective and objective analysis with the method of entropy weight. It can not only make the weight of the evaluation model objective, but also avoid the difficulty of using the objective method to determine the weight in the absence of data.

\subsubsection{Analytic Hierarchy Process}

The Analytic Hierarchy Process (AHP) decomposes a complex decision-making problem into multiple objectives or criteria. Then the hierarchical single ranking and total ranking are calculated by using the qualitative index fuzzy quantization method. It can be used as a systematic method for multi-scheme optimization decision-making $[6,8,12]$. The steps of the analytic hierarchy process are as follows.

1. Establishing hierarchical structure model.

2. Construct judgement matrices at all levels.

$$
C=\left[\begin{array}{cccc}
C_{11} & C_{12} & \cdots & C_{1 n} \\
C_{21} & C_{22} & \cdots & C_{2 n} \\
\vdots & \vdots & \ddots & \vdots \\
C_{n 1} & C_{n 2} & \cdots & C_{n n}
\end{array}\right]
$$

In Equation (12), $C_{i j}=1 / C_{j i}$. The assignment of $C_{i j}$ is shown in Table 2. 
Table 2. $C_{i j}$ value table.

\begin{tabular}{cl}
\hline Value & \multicolumn{1}{c}{ Meaning } \\
\hline 1 & The two factors are equally important. \\
3 & The former is slightly more important than the latter. \\
7 & The former is more important than the latter. \\
9 & The former is strongly more important than the latter. \\
$2,4,6,8$ & The former is extremely more important than the latter. \\
\hline
\end{tabular}

3 Calculating subjective weights. After the judgment matrix $C$ is established, the maximum eigenvalue $\lambda_{\max }$ of the judgment matrix $C$ is obtained. Then the eigenvector of $\lambda_{\max }$ is obtained. After normalization, the subjective weight $W=\left(w_{1}, \ldots, w_{n}\right)^{T}$ is obtained.

4 Consistency test. The consistency index $C R$ of judgment matrix can be expressed as the following equation.

$$
\begin{gathered}
C R=\frac{C I}{R I^{\prime}} \\
C I=\frac{\lambda_{\max }-n}{n-1} .
\end{gathered}
$$

In Equations (13) and (14), $C I$ is the consistency indicator. $n$ is the order of the judgment matrix. $R I$ is the random consistency ratio of the judgment matrix. Its assignment is shown in Table 3 . When $C R<0.1$, the judgment matrix has satisfactory consistency. Otherwise, the judgment matrix should be readjusted until it has satisfactory consistency.

Table 3. $R I$ value table.

\begin{tabular}{cccccccccccc}
\hline$N$ & $\mathbf{1}$ & $\mathbf{2}$ & $\mathbf{3}$ & $\mathbf{4}$ & $\mathbf{5}$ & $\mathbf{6}$ & $\mathbf{7}$ & $\mathbf{8}$ & $\mathbf{9}$ & $\mathbf{1 0}$ & $\mathbf{1 1}$ \\
$R I$ & 0 & 0 & 0.58 & 0.90 & 1.12 & 1.24 & 1.32 & 1.41 & 1.45 & 1.49 & 1.51 \\
\hline
\end{tabular}

Let $C I(j)$ be the consistency index of the judgment matrix of the factors related to the upper level in layer $\mathrm{B}$. The corresponding average random consistency index is $R I(j)$. Then the random consistency ratio of the B-level total ranking is shown in the following Equation (15).

$$
C R=\frac{\sum_{j=1}^{m} C I(j) a_{j}}{\sum_{j=1}^{m} R I(j) a_{j}} .
$$

When $C R<0.1$, it is considered that the results are in good agreement and the analysis results are acceptable.

\subsubsection{Entropy Weight Method}

The basic idea of the entropy weight method is to determine the objective weight according to the variability of the index. Generally speaking, the smaller the information entropy of an index, the greater the variation degree of the index. The more information it provides, the greater the role it can play in the comprehensive evaluation, and the greater its weight. On the contrary, the greater the information entropy of an index, the smaller the variation degree of the index value. The less information it provides, the smaller the role it plays in the comprehensive evaluation, and the smaller its weight. The entropy weight method makes effective use of index data, excluding the influence of subjective factors $[13,16]$. The steps of the entropy weight method are as follows.

1. Data standardization. 
Suppose there are $n$ evaluation objects, $m$ indicators $\left\{X_{1}, X_{2}, \ldots, X_{m}\right\}$, where $X_{i}=\left\{x_{1}, x_{2}, \ldots, x_{n}\right\}$. Assume that the normalized values for each data are $\left\{Y_{1}, Y_{2}, \ldots, Y_{m}\right\}$. Indicators are divided into positive indicators and negative indicators. The positive index refers to the index that the larger the value, the better the result. Negative indicators refer to indicators that the smaller the value, the better the result. In order to keep the consistency of each index in information entropy, data processing should be separated.

For positive indicators:

$$
Y_{i j}=\frac{x_{i j}-\min \left(X_{i}\right)}{\max \left(X_{i}\right)-\min \left(X_{i}\right)} .
$$

For negative indicators:

$$
Y_{i j}=\frac{\max \left(X_{i}\right)-x_{i j}}{\max \left(X_{i}\right)-\min \left(X_{i}\right)} .
$$

2 Calculate the information entropy of each index. According to the definition of information entropy in information theory, the information entropy $e_{j}$ of a group of data is shown in the following equation.

$$
\begin{gathered}
e_{j}=-\sum_{i=1}^{n} p_{i j} \ln p_{i j} / \ln n, \\
p_{i j}=Y_{i j} / \sum_{i=1}^{n} Y_{i j} .
\end{gathered}
$$

If $p_{i j}=0$, then the definition $\lim _{p_{i j} \rightarrow 0} p_{i j} \ln p_{i j}=0$.

3 Calculate the coefficient of difference of $j$ index.

$$
g_{j}=1-e_{j}
$$

The greater the difference coefficient $g_{j}$ is, the greater the effect of this index is.

4 Calculate objective weight $v_{j}$ of index $j$.

$$
v_{j}=g_{j} / \sum_{i=1}^{m} g_{j} .
$$

\subsubsection{Comprehensive Weight}

Generally, the results obtained by subjective and objective methods are different. In order to make the results more accurate, many studies use the method of comprehensive weights to synthesize weights. Most studies on the assignment of comprehensive weights are the sum of the two [25-27] or the direct multiplication of the two [28,29]. In this paper, the principle of minimum information discrimination is used to optimize the proportion of subjective and objective weights so as to make the evaluation results more accurate. Let the subjective weight vector $\alpha$ and the objective weight vector $\beta$ be obtained by the above analytic hierarchy process and the entropy weight method respectively. In order to make the comprehensive weights as close as possible to $\alpha$ and $\beta$, the comprehensive weights $\omega_{i}$ are calculated according to the principle of minimum discriminant information. The objective function is shown in the following Equation (22).

$$
\left\{\begin{array}{c}
\min J(\omega)=\sum_{i=1}^{m}\left(\omega_{i} \ln \frac{\omega_{i}}{\alpha_{i}}+\omega_{i} \ln \frac{\omega_{i}}{\beta_{i}}\right) \\
\text { s.t. } \sum_{i=1}^{m} \omega_{i}=1, \omega_{i} \geq 0 \quad i=1,2, \cdots, m
\end{array} .\right.
$$


Using the Lagrange multiplier method to solve the optimization model [30], the comprehensive weight is obtained as shown in the following Equation (23).

$$
\omega_{i}=\frac{\sqrt{\alpha_{i} \beta_{i}}}{\sum_{j=1}^{m} \sqrt{\alpha_{i} \beta_{i}}} .
$$

\subsection{Evaluation Method Combining Comprehensive Weight and Grey Relational Degree Analysis}

Grey relational degree analysis shows that the closer the geometric shapes of the curves formed by several statistical series are, that is, the more parallel the curves are, the closer their changing trend is, and the greater their relational degree is. Therefore, it is possible to compare and rank the evaluation objects by using the correlation degree between the schemes and the optimal schemes. The method first obtains the correlation coefficient matrix between each scheme and the ideal scheme, and then obtains the correlation degree from the correlation coefficient matrix. Finally, rank and analyze according to the size of correlation degree, and draw a conclusion [31]. The steps of grey correlation analysis are as follows.

1. According to the analysis purpose, the analysis index system is determined, and the analysis data are collected. Let the data sequence form the following matrix:

$$
\left(X_{1}^{\prime}, X_{2}^{\prime}, \cdots X_{m}^{\prime}\right)=\left(\begin{array}{cccc}
x_{1}^{\prime}(1) & x_{2}^{\prime}(1) & \cdots & x_{m}^{\prime}(1) \\
x_{1}^{\prime}(2) & x_{2}^{\prime}(2) & \cdots & x_{m}^{\prime}(2) \\
\vdots & \vdots & \ddots & \vdots \\
x_{1}^{\prime}(n) & x_{2}^{\prime}(n) & \cdots & x_{m}^{\prime}(n)
\end{array}\right)
$$

Among them, $m$ is the number of indicators and $n$ is the number of samples. $X_{i}^{\prime}(1)=\left(x_{i}^{\prime}(1), x_{i}^{\prime}(2)\right.$, $\left.\ldots, x_{i}^{\prime}(n)\right)^{T}, i=1,2, \ldots, n$.

2 Data standardization

For positive indicators, the process is the same as Equation (16). For negative indicators, the process is the same as Equation (17).

3 Computation of correlation coefficient

The correlation coefficients of the elements corresponding to each comparison sequence and the reference sequence are calculated respectively.

$$
\begin{gathered}
\zeta_{i}(k)=\frac{\Delta \min +\rho \cdot \Delta \max }{\left|x_{0}(k)-x_{i}(k)\right|+\rho \cdot \Delta \max }, k=1, \cdots, m, \\
\Delta \min =\min _{i=1}^{n} \min _{k=1}^{m}\left|x_{0}(k)-x_{i}(k)\right|, \Delta \max =\operatorname{maxmax}_{i=1}^{n}\left|x_{0}(k)-x_{i}(k)\right| .
\end{gathered}
$$

Among them, $\zeta_{i}(k)$ is the correlation coefficient of $k$ indicator between ideal scheme $x_{0}$ and scheme $x_{i} . \rho$ is the resolution coefficient, $0<\rho<1$. The smaller the $\rho$, the greater the difference between correlation coefficients and the stronger the discrimination ability. Usually we take $\rho=0.5 . x_{0}$ is the ideal solution.

\section{Computation of correlation degree}

The correlation degree between $i$ scheme and ideal scheme is as follows.

$$
\gamma_{i}=\frac{1}{m} \sum_{k=1}^{m} \omega_{k} \cdot \zeta_{i}(k)(k=1, \cdots, m)
$$


In Equation (26), $\omega_{k}$ is the comprehensive weight of each index.

Finally, the optimal scheme is selected according to the value of correlation degree $\gamma_{i}$.

\subsection{Summary}

The flow chart of the comprehensive evaluation model of AC/DC hybrid microgrid planning based on AHP and entropy weight method is shown in Figure 2.

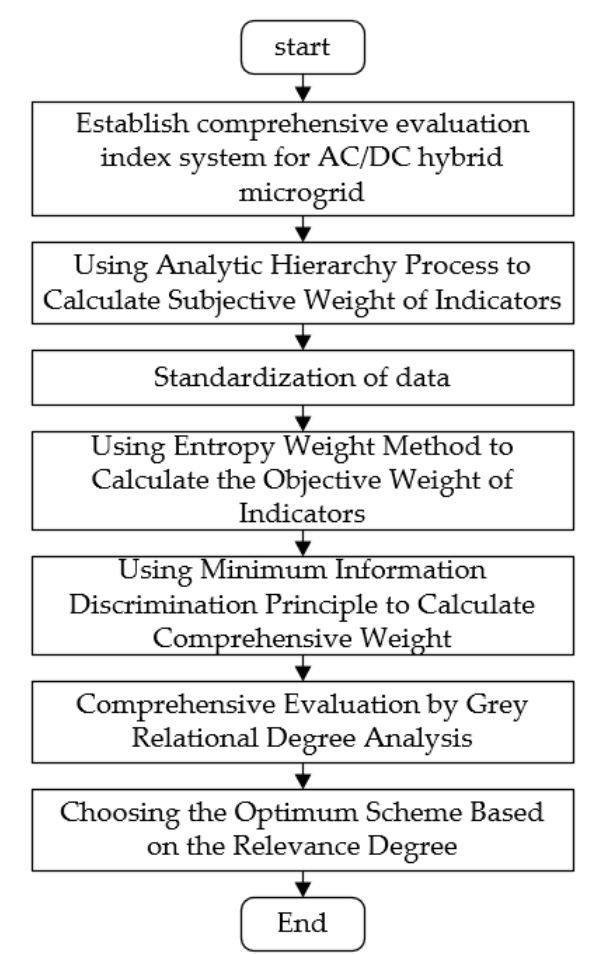

Figure 2. Comprehensive evaluation flow of AC/DC hybrid microgrid planning based on analytic hierarchy process and entropy weight method.

The scientific and reasonable evaluation index decides whether the evaluation is reasonable or not. Before comprehensive evaluation, this paper had a comprehensive and in-depth understanding of the planning of an AC/DC hybrid microgrid. An objective, reasonable, and scientific evaluation index system for AC/DC hybrid microgrid planning is established on the basis of searching a large number of documents and materials.

After determining the index system, the corresponding weight of each index should be determined. In this paper, a combination of subjective and objective methods is used. On the basis of determining the weights of AHP and entropy weight, the weights are combined by the principle of minimum information discrimination. This not only reduces the subjective uncertainty and cognitive ambiguity of the analytic hierarchy process, but also reduces the one-sidedness caused by relying solely on sample data and the contingency caused by incomplete sample data. It not only effectively absorbs the results of qualitative analysis, but also gives full play to the advantages of quantitative analysis.

Finally, the comprehensive evaluation of indicators is carried out by combining the grey relational degree analysis method. Equation (26) gives the comprehensive evaluation results combined with comprehensive weight and grey relational degree analysis method. After the value of $\gamma_{i}$ is obtained, the optimal scheme is determined by ranking the correlation degree between each scheme and the ideal scheme. Through the above process, this paper establishes a comprehensive evaluation model of AC/DC hybrid microgrid planning based on analytic hierarchy process and entropy weight method. 


\section{Example Analysis}

Taking the following four AC/DC hybrid microgrid schemes as examples, the comprehensive evaluation method of planning for AC/DC hybrid microgrid proposed in this paper is analyzed and calculated. The schematic structure of each scheme is shown in Figure 3. The parameters of each scheme are shown in Table 4.

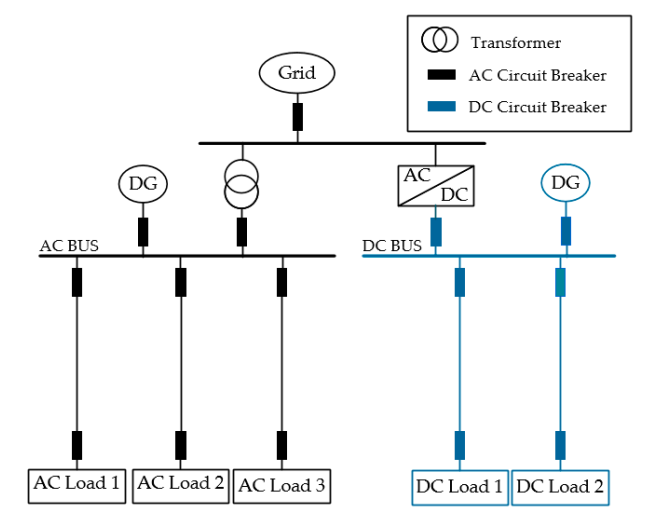

(a) Scheme 1 Structural Diagram.

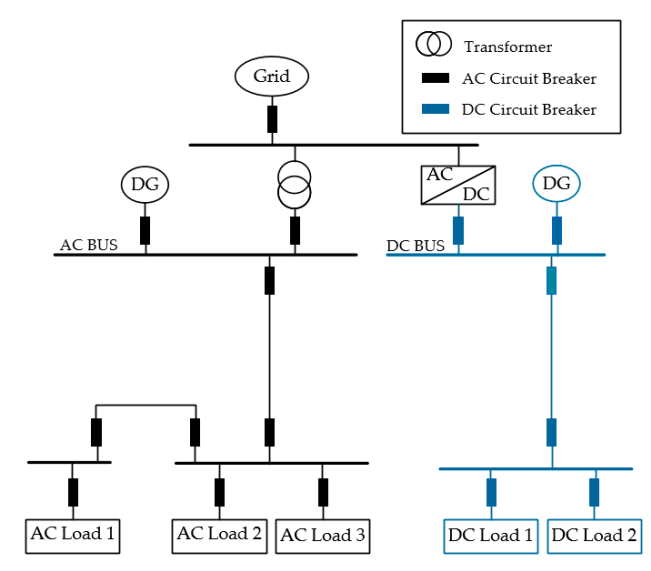

(b) Scheme 2 Structural Diagram

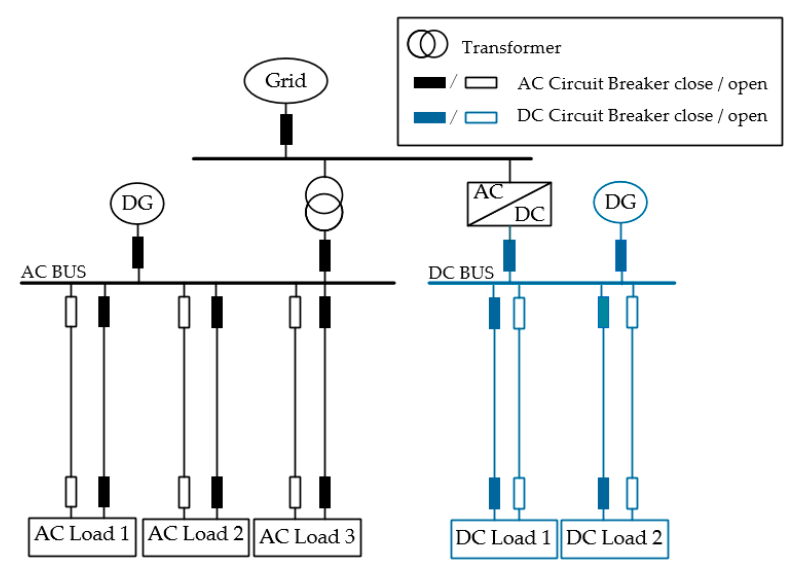

(c) Scheme 3 Structural Diagram

Figure 3. Cont. 


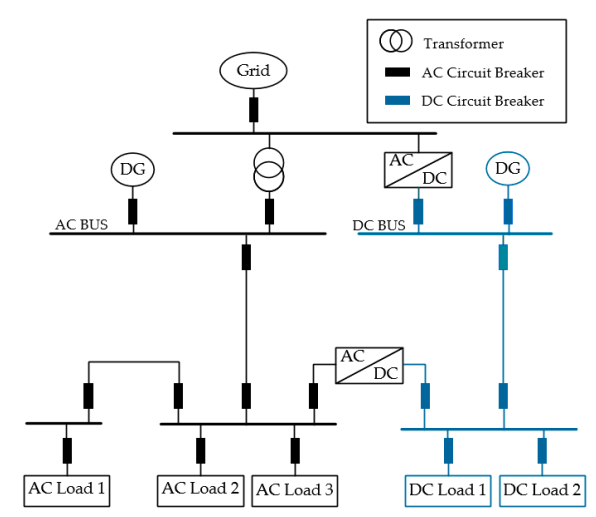

(d) Scheme 4 Structural Diagram

Figure 3. Power grid structure diagram.

Table 4. Scheme parameters.

\begin{tabular}{|c|c|c|c|c|c|}
\hline First-Level Indicators & Secondary Indicators & Scheme 1 & Scheme 2 & Scheme 3 & Scheme 4 \\
\hline \multirow{2}{*}{$\begin{array}{l}\text { The reliability indicators } \\
\text { (A1) }\end{array}$} & $\begin{array}{l}\text { Average service availability } \\
\text { index (B1)/\% }\end{array}$ & 99.9784 & 99.9630 & 99.9911 & 99.9863 \\
\hline & $\begin{array}{l}\text { System average interruption } \\
\text { duration index (B2)/(h/year) }\end{array}$ & 1.892 & 3.241 & 0.780 & 1.200 \\
\hline \multirow{3}{*}{$\begin{array}{l}\text { The economic indicators } \\
\text { (A2) }\end{array}$} & $\begin{array}{l}\text { Initial investment cost (B3)/ } \\
\text { (RMB 10,000) }\end{array}$ & 6270 & 5982 & 7990 & 7476 \\
\hline & $\begin{array}{l}\text { Operation and maintenance cost } \\
\text { (B4)/(RMB 10,000) }\end{array}$ & 31.35 & 29.91 & 39.95 & 37.38 \\
\hline & $\begin{array}{l}\text { Network loss cost (B5)/ } \\
(\text { RMB 10,000) }\end{array}$ & 15.768 & 11.038 & 15.768 & 12.614 \\
\hline \multirow{3}{*}{$\begin{array}{l}\text { The power quality } \\
\text { indicators (A3) }\end{array}$} & Voltage offset (B6)/\% & 0.42 & 0.51 & 0.42 & 0.33 \\
\hline & Frequency deviation (B7) & Excellent & Good & Excellent & Excellent \\
\hline & Harmonic distortion rate (B8) & Good & Fair & Good & Excellent \\
\hline \multirow{2}{*}{$\begin{array}{l}\text { The environmental } \\
\text { Protection Indicators(A4) }\end{array}$} & Emissions of polluted gases (B9) & Excellent & Good & Excellent & Excellent \\
\hline & Land occupation area $(\mathrm{B} 10) / \mathrm{m}^{2}$ & 2420.5 & 2401.42 & 2487.1 & 2442.2 \\
\hline
\end{tabular}

\subsection{Using Analytic Hierarchy Process (AHP) to Calculate Subjective Weight}

The first-level indicator judgment matrix is as follows:

$$
C_{1}=\left(\begin{array}{cccc}
1 & 1 & 1 & 2 \\
1 & 1 & 1 & 2 \\
1 & 1 & 1 & 3 \\
1 / 2 & 1 / 2 & 1 / 3 & 1
\end{array}\right)
$$

The calculation results show that $\lambda_{1 \max }=4.0206$. The corresponding weight vector is [0.2804, $0.2804,0.3120,0.1272]^{T} . C R_{1}=0.0076<0.1$. It passes consistency verification.

The judgment matrix of the secondary indicator corresponding to reliability is as follows:

$$
C_{2}=\left(\begin{array}{cc}
1 & 2 \\
1 / 2 & 1
\end{array}\right)
$$

The calculation results show that $\lambda_{2 \max }=2.0000$. The corresponding weight vector is [0.6667, $0.3333]^{T} . C R_{2}=0<0.1$. It passes consistency verification. 
The judgment matrix of the secondary indicator corresponding to economy is as follows:

$$
C_{3}=\left(\begin{array}{ccc}
1 & 2 & 3 \\
1 / 2 & 1 & 2 \\
1 / 3 & 1 / 2 & 1
\end{array}\right)
$$

The calculation results show that $\lambda_{3 \max }=3.0092$. The corresponding weight vector is [0.5396, $02970,0.1634]^{T} . C R_{3}=0.0046<0.1$. It passes consistency verification.

The judgment matrix of the secondary indicator corresponding to power quality is as follows:

$$
C_{4}=\left(\begin{array}{ccc}
1 & 1 & 3 \\
1 & 1 & 3 \\
1 / 3 & 1 / 3 & 1
\end{array}\right)
$$

The calculation results show that $\lambda_{4 \max }=3.0000$. The corresponding weight vector is $[0.4286$, $0.4286,0.1429]^{T}$. $C R_{4}=0<0.1$. It passes consistency verification.

The judgment matrix of the secondary indicator corresponding to environmental protection is as follows:

$$
C_{5}=\left(\begin{array}{cc}
1 & 2 \\
1 / 2 & 1
\end{array}\right)
$$

The calculation results show that $\lambda_{5 \max }=2.0000$. The corresponding weight vector is $[0.6667$, $0.3333]^{T} . C R_{5}=0<0.1$. It passes consistency verification.

Using the Equation (15), we can check the consistency of hierarchical total ranking. $C R=0.0046<$ 0.1 . It passes consistency verification. Therefore, the subjective weight of each evaluation indicator is

\begin{tabular}{|c|c|c|c|c|}
\hline $\begin{array}{l}\text { First-Level } \\
\text { Indicators }\end{array}$ & Weight & Secondary Indicators & $\begin{array}{c}\text { The Weights of } \\
\text { Layer A }\end{array}$ & Subjective Weight \\
\hline \multirow[b]{2}{*}{$\begin{array}{l}\text { The reliability } \\
\text { indicators (A1) }\end{array}$} & \multirow[b]{2}{*}{0.2804} & $\begin{array}{c}\text { Average service } \\
\text { availability index (B1) }\end{array}$ & 0.6667 & 0.1869 \\
\hline & & $\begin{array}{l}\text { System average } \\
\text { interruption duration } \\
\text { index (B2) }\end{array}$ & 0.3333 & 0.0935 \\
\hline \multirow{3}{*}{$\begin{array}{l}\text { The economic } \\
\text { indicators (A2) }\end{array}$} & \multirow{3}{*}{0.2804} & $\begin{array}{l}\text { Initial investment } \\
\text { cost (B3) }\end{array}$ & 0.5396 & 0.1513 \\
\hline & & $\begin{array}{c}\text { Operation and } \\
\text { maintenance cost (B4) }\end{array}$ & 0.2970 & 0.0833 \\
\hline & & Network loss cost (B5) & 0.1634 & 0.0458 \\
\hline \multirow{3}{*}{$\begin{array}{l}\text { The power quality } \\
\text { indicators (A3) }\end{array}$} & \multirow{3}{*}{0.3120} & Voltage offset (B6) & 0.4286 & 0.1337 \\
\hline & & $\begin{array}{c}\text { Frequency } \\
\text { deviation (B7) }\end{array}$ & 0.4286 & 0.1337 \\
\hline & & $\begin{array}{c}\text { Harmonic distortion } \\
\text { rate }(\mathrm{B} 8)\end{array}$ & 0.1429 & 0.0446 \\
\hline \multirow{2}{*}{$\begin{array}{l}\text { The environmental } \\
\text { protection } \\
\text { indicators (A4) }\end{array}$} & \multirow{2}{*}{0.1272} & $\begin{array}{c}\text { Emissions of polluted } \\
\text { gases (B9) }\end{array}$ & 0.6667 & 0.0848 \\
\hline & & $\begin{array}{l}\text { Land occupation area } \\
\text { (B10) }\end{array}$ & 0.3333 & 0.0424 \\
\hline
\end{tabular}
shown in Table 5 and Figure 4.

Table 5. Subjective weight obtained by analytic hierarchy process. 


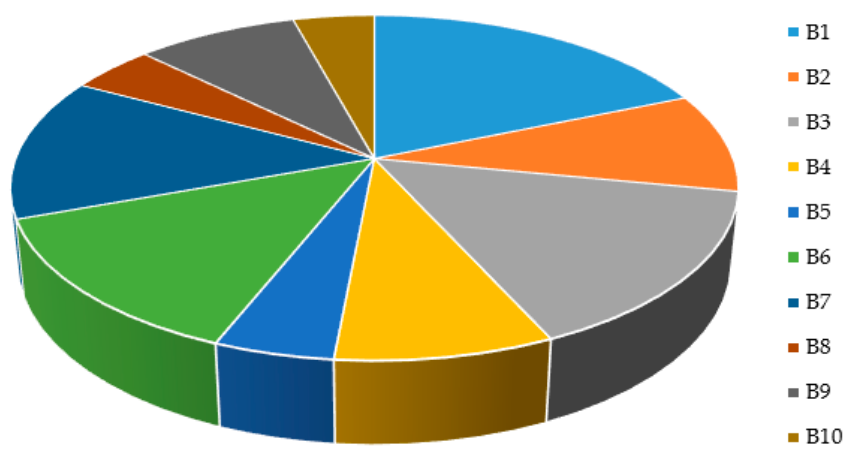

Figure 4. The subjective weight of each evaluation indicator.

\subsection{Using Entropy Weight Method to Calculate Objective Weight}

Firstly, data are standardized by Equations (16) and (17). Individual indicators in the table lack sufficient data. Quantitative evaluation criteria were designed in a five-point scale rating scale, that is excellent, good, fair, qualified and unqualified. In this paper, $\{5,4,3,2,1\}$ is used to denote excellent, good, fair, qualified, unqualified\} respectively. The standardized data and weights of each evaluation index are shown in Table 6.

Table 6. Standardized data and objective weights.

\begin{tabular}{|c|c|c|c|c|c|}
\hline Secondary Indicators & Scheme 1 & Scheme 2 & Scheme 3 & Scheme 4 & $\begin{array}{c}\text { Objective } \\
\text { Weight }\end{array}$ \\
\hline Average service availability index (B1) & 0.5480 & 0 & 1 & 0.8292 & 0.0842 \\
\hline $\begin{array}{l}\text { System average interruption duration } \\
\text { index (B2) }\end{array}$ & 0.5480 & 0 & 1 & 0.8292 & 0.0842 \\
\hline Initial investment cost (B3) & 0.8566 & 1 & 0 & 0.2560 & 0.1093 \\
\hline Operation and maintenance cost (B4) & 0.8566 & 1 & 0 & 0.2560 & 0.1093 \\
\hline Network loss cost (B5) & 0 & 1 & 0 & 0.6667 & 0.1899 \\
\hline Voltage offset (B6) & 0.5 & 0 & 0.5 & 1 & 0.0923 \\
\hline Frequency deviation (B7) & 1 & 0 & 1 & 1 & 0.0766 \\
\hline Harmonic distortion rate (B8) & 0.5 & 0 & 0.5 & 1 & 0.0923 \\
\hline Emissions of polluted gases (B9) & 1 & 0 & 1 & 1 & 0.0766 \\
\hline Land occupation area (B10) & 0.7773 & 1 & 0 & 0.5243 & 0.0853 \\
\hline
\end{tabular}

The objective weights calculated by the entropy method are shown in the Figure 5 .

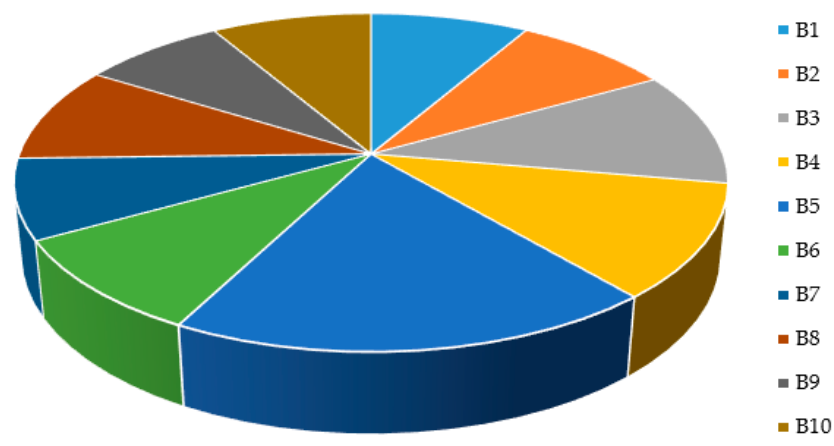

Figure 5. The objective weights of each evaluation indicator.

\subsection{Determining the Comprehensive Weight}

Equation (23) is used to obtain the comprehensive weights of each evaluation index as shown in Table 7 and Figure 6. Figure 7 is a weight map of each evaluation index. 
Table 7. Comprehensive weight.

\begin{tabular}{cccc}
\hline Secondary Indicators & Subjective Weight & Objective Weight & Comprehensive Weight \\
\hline Average service availability index (B1) & 0.1869 & 0.0842 & 0.1323 \\
System average interruption duration index (B2) & 0.0935 & 0.0842 & 0.0935 \\
Initial investment cost (B3) & 0.1513 & 0.1093 & 0.1356 \\
Operation and maintenance cost (B4) & 0.0833 & 0.1093 & 0.1006 \\
Network loss cost (B5) & 0.0458 & 0.1899 & 0.0983 \\
Voltage offset (B6) & 0.1337 & 0.0923 & 0.1171 \\
Frequency deviation (B7) & 0.1337 & 0.0766 & 0.1067 \\
Harmonic distortion rate (B8) & 0.0446 & 0.0923 & 0.0676 \\
Emissions of polluted gases (B9) & 0.0848 & 0.0766 & 0.0850 \\
Land occupation area (B10) & 0.0424 & 0.0853 & 0.0634 \\
\hline
\end{tabular}

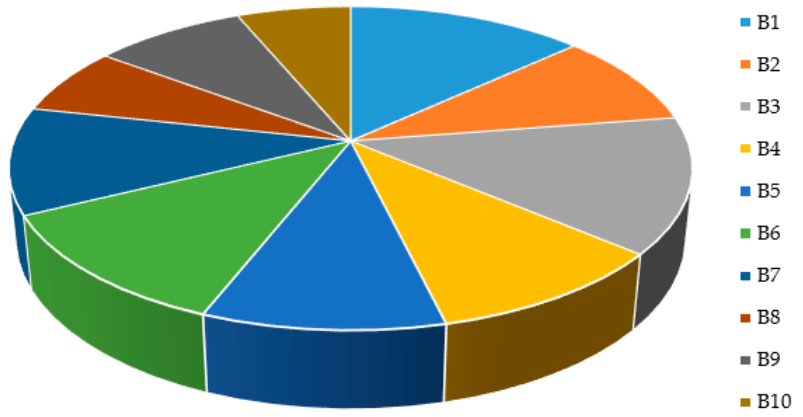

Figure 6. The objective weights of each evaluation indicator.

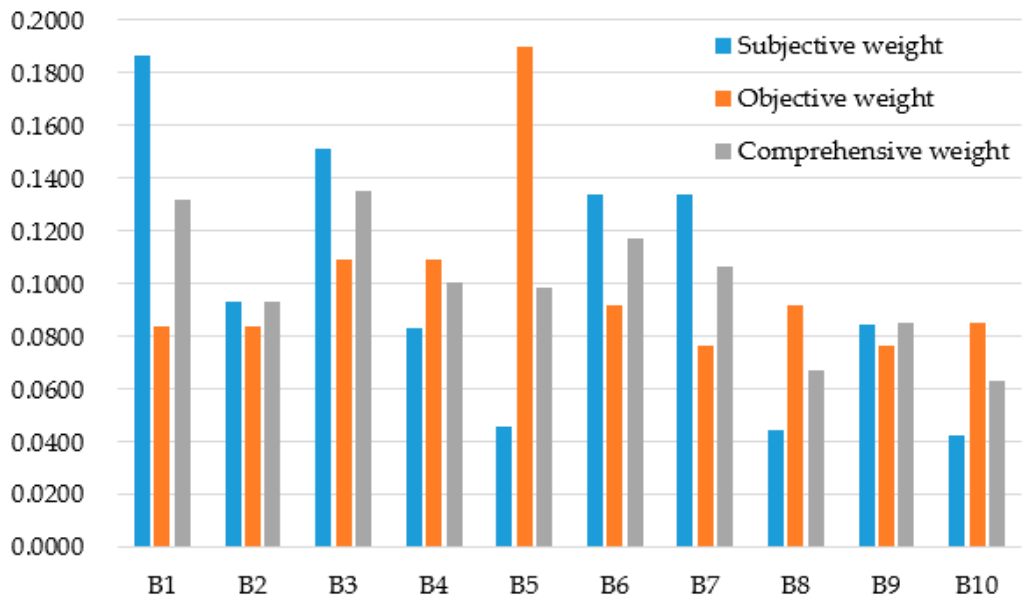

Figure 7. The weights of each evaluation indicator.

\subsection{Applying Grey Relational Degree Analysis Method to Evaluate and Find the Optimum Scheme}

Based on the data in Table 6 , we define the best scenario as $x_{0}=[1,1,1,1,1,1,1,1,1,1]^{T}$. The correlation coefficients of four schemes under each index are obtained by Equation (25) as shown in Figure 8. Then the comprehensive weight and correlation coefficient calculated above are substituted into Equation (26) to calculate the correlation degree of the four schemes as shown in Table 8. 


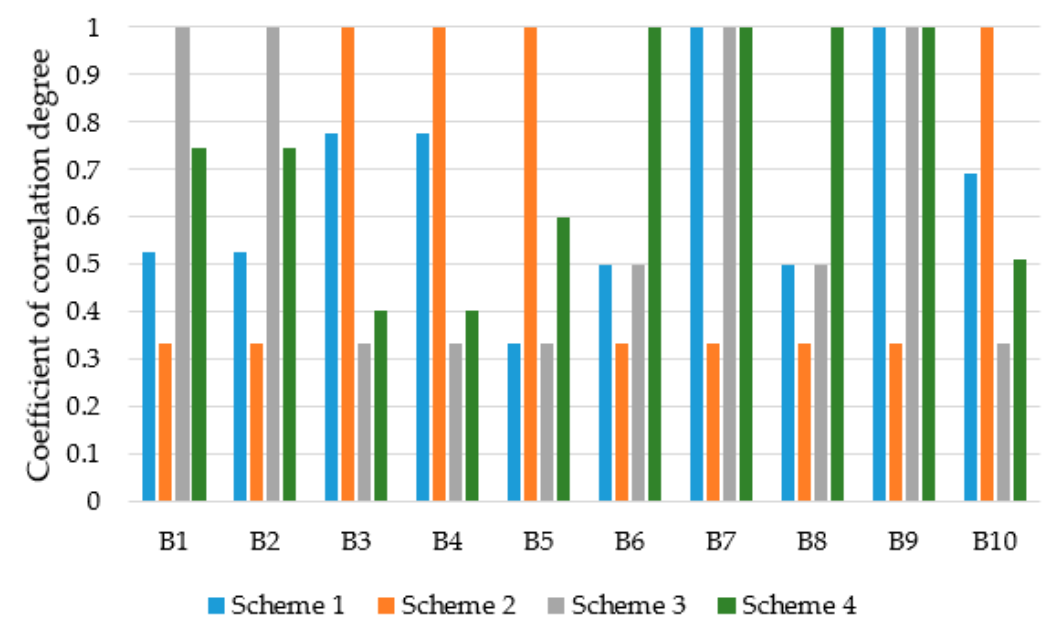

Figure 8. Relevance coefficients of various schemes under various indicators.

Table 8. Ranking of schemes.

\begin{tabular}{ccccc}
\hline Schemes & Scheme 1 & Scheme 2 & Scheme 3 & Scheme 4 \\
\hline Correlation degree $\gamma$ & 0.6627 & 0.5986 & 0.6424 & 0.7310 \\
Ranking & 4 & 2 & 3 & 1 \\
\hline
\end{tabular}

From Table 8 , we can see that scheme 4 is the best scheme and scheme 2 is the worst scheme.

\section{Discussion}

In Figure 4 and Table 5, we obtained the subjective weights of each index calculated by analytic hierarchy process. Among them, the subjective weights of B1, B3, B6, and B7 are larger, while the subjective weights of B5, B8, and B10 are smaller. In Figure 5 and Table 6, we obtained the objective weights of each index calculated by the method of entropy weight. Among them, the objective weights of B3, B4, and B5 are larger, while the objective weights of B1, B2, B7, and B9 are smaller. There are some differences between subjective weight and objective weight, which is caused by their different characteristics. Subjective methods are more inclined to people's experience, while objective methods depend entirely on numerical values, so the results of the two methods will be different or even conflict.

In Figure 7 and Table 7, we obtained the comparison of subjective weight, objective weight, and comprehensive weight. Among them, the comprehensive weights of B1, B3, and B6 are larger, while the comprehensive weights of B8 and B10 are smaller. We can see that the weight of the B7 index is larger when the subjective evaluation method determines the weight, while the weight of the B7 index is not so obvious in the comprehensive weight. The reason is that the B7 index is given a greater weight in the calculation of AHP is because of the human experience deviation. This paper corrects the human experience error by combining objective methods. The weight of the B5 index is very large when the objective evaluation method determines the weight, but the weight of the B5 index is not so obvious in the comprehensive weight. The reason is that the B5 index has a large error when it is calculated by using the method of entropy weight because of the insufficient amount of data. This paper combines the subjective method to correct the data error. From the Figure 7, it can be seen that the comprehensive weight of each index is between that determined by the subjective method and objective method. The comprehensive weights obtained by the minimum information discriminant principle can overcome the shortcomings of the analytic hierarchy process (AHP) and the entropy weight method. The comprehensive weight has the advantages of both subjective weight and objective weight. It can make full use of the existing data and reduce the large errors caused by inadequate or inaccurate raw data by combining subjective experience.

In Figure 8, we obtained the correlation degree between the four schemes and the ideal scheme under each index calculated by grey correlation analysis method. The correlation degree of each 
index of scheme 4 is higher. The correlation degree of some indicators in scheme 1 and scheme 3 is higher, and that of individual indicators is lower. The correlation degree of most of the indicators in scheme 2 is lower. In Table 8, we can see the correlation degree and ranking of four schemes with the ideal scheme. Scheme 4 is the best scheme, and scheme 2 is the worst one. In practice, scheme 4 has high reliability, high power quality, and good environmental protection. Scheme 2 has the lowest reliability and poor power quality although it has low cost. Scheme 1 is moderate in all aspects and has no obvious advantages and disadvantages. Scheme 3 has high reliability, but its cost is very high. The final evaluation results are consistent with the actual situation. This result reflects the correctness and rationality of the comprehensive evaluation method in this paper. Generally speaking, the evaluation method used in this paper conforms to the actual engineering effect and has certain social application value.

\section{Conclusions}

Aiming at comprehensive evaluation of AC/DC hybrid microgrid planning, a new evaluation index system of $\mathrm{AC} / \mathrm{DC}$ hybrid microgrid is constructed in the paper. The new evaluation index system of AC/DC hybrid microgrid planning is comprehensive, scientific, and accurate, which provides a new way to study the evaluation index of AC/DC hybrid microgrid planning.

Aiming at the limitations of traditional evaluation methods, a comprehensive evaluation method of AC/DC hybrid microgrid planning based on analytic hierarchy process (AHP) and entropy weight method is proposed. The method is divided into three parts: the establishment of the evaluation index system, the determination of comprehensive weight, and the comprehensive evaluation of the scheme. The comprehensive evaluation method presented in this paper provides a new idea for the comprehensive evaluation of AC/DC hybrid microgrid planning in the future.

From summarizing the theoretical analysis, the model establishment, and the verification of simulation examples in this paper, the following conclusions can be drawn:

1. In the example of this paper, scheme 4 is the optimal scheme, which can provide a research idea for the future planning of AC/DC hybrid microgrids.

2. Compared with traditional methods, the comprehensive evaluation method proposed in this paper has the advantages of objective evaluation and subjective evaluation, which can reduce the errors caused by the subjective evaluation method being more subjective and the objective method being too dependent on data. The evaluation method in this paper has certain practical application value.

3. The results of examples in this paper show that the average service availability index, initial investment cost, and voltage offset are the three most important indicators in the comprehensive evaluation index system. The reason is that their comprehensive weight is relatively large. Therefore, people should pay more attention to these three indicators in the design of AC/DC hybrid microgrids.

Author Contributions: Conceptualization, G.Z.; methodology, G.Z., D.W.; software, D.W.; validation, G.Z., D.W.; formal analysis, D.W.; investigation and resources, D.W.; data curation, D.W.; writing-original draft preparation, G.Z., D.W.; writing - review and editing, D.W.; supervision, G.Z., D.W.; project administration, G.Z.

Funding: This research was funded by the Fundamental Research Funds for the Central Universities (2018MS008).

Conflicts of Interest: The authors declare no conflict of interest.

\section{References}

1. Adam, H.; Yael, P.; Josep, G. Microgrids: A review of technologies, key drivers, and outstanding issues. Renew. Sustain. Energy Rev. 2018, 90, 402-411.

2. Esther, M.; Johannes, G.; Kerstin, R. Designing microgrid energy markets A case study: The Brooklyn Microgrid. Appl. Energy 2018, 210, 870-880. 
3. Chen, Z.; Zheng, T.; Liu, C. An Islanding Signal-Based Smooth Transition Control in AC/DC Hybrid Micro-Grids. Appl. Sci. 2019, 9, 2804. [CrossRef]

4. Chen, J.; Chen, C.; Duan, S. Cooperative Optimization of Electric Vehicles and Renewable Energy Resources in a Regional Multi-Microgrid System. Appl. Sci. 2019, 9, 2267. [CrossRef]

5. Liu, H.-M.; Huang, C.-Y.; Chen, Y. Vulnerability Assessment for Microgrid with Risk Theory. Proc. CSU EPSA 2014, 26, 1-6.

6. Mu, Y.; Lu, Z.; Qiao, Y.; Wang, Y.; Huang, H.; Zhou, Q.; Han, J. A Comprehensive Evaluation Index System of Power Grid Security and Benefit Based on Multi-Operator Fuzzy Hierarchy Evaluation Method. Power Syst. Technol. 2015, 39, 23-28.

7. Liu, Z.; Wei, T.; Li, M.; Cao, Z.; Li, W.; Hui, H. Comprehensive evaluation based on improved analytic hierarchy process for AC/DC microgrid. Electr. Power Autom. Equip. 2016, 36, 60-66.

8. Jiang, G.; Wang, Z.; Suo, Y. Hierarchical analysis and fuzzy evaluation of comprehensive performance of typical water-saving irrigation techniques in Northwest China. J. Tsinghua Univ. (Sci. Technol.) 2019, 1-9. [CrossRef]

9. Liu, M.; Zhao, L.; Huang, L.; Lin, C.; Su, H.; Long, Z.; Liu, Y.; Zhang, S.; Zhang, X. Power grid risks assessment and classification based on fuzzy comprehensive evaluation. Eng. J. Wuhan Univ. 2017, 50, 733-737.

10. Dai, X.; Wang, Y.; Bai, C.; Yang, F.; Jin, X.; Zhou, J.; Yu, J.; Zhao, L. Analysis Framework and Fuzzy Comprehensive Evaluation of Smart Grid Function and Form Upgrade Needs. Electr. Power 2017, 50, 158-164.

11. Liu, J. Study on Comprehensive Evaluation of Utilization Ratio of Shenzhen Power Grid Equipment Based on Analytic Hierarchy Process Method. Ph.D. Thesis, South China University of Technology, Guangzhou, China, 2018.

12. Yang, B.; Zhao, J.; Tang, W.; Fang, Y. Research on the Evaluation Method of Power Grid Science and Technology Project based on Analytic Hierarchy Process. Electr. Eng. 2017, 90-94. [CrossRef]

13. Qi, M.; Fu, Z.; Jing, Y.; Ma, Y. A Comprehensive Evaluation Method of Power Plant Units Based on Information Entropy and Principal Component Analysis. Proc. CSEE 2013, 33, 58-64.

14. Zong, X. Study and Analysis on Power Grid Operation State Characteristics Based on Entropy Weight Method. Ph.D. Thesis, Tianjin University, Tianjin, China, 2017.

15. Wu, H.; An, S.; Chai, J.; Zhou, Y. Application of Set Pair Model to Substation Project Post-Evaluation Based on Entropy Weight. Power Energy 2018, 39, 49-52.

16. Xiong, W.; Zhang, H.; Xie, Z.; Han, X.; Li, Z.; Lin, X. Risk assessment of power transformer potential fault based on cloud theory and entropy weight method. Electr. Power Autom. Equip. 2018, 38, 125-130.

17. Liang, H.; Tian, S.; Li, Q.; Liu, Y.; Zhang, W. Main grid structure evaluation based on improved TOPSIS grey relation projection method. Electr. Power Autom. Equip. 2019, 39, 63-69.

18. Chang, Y.; Chen, Y. G1 Method on the Basis of Index Number Scale and Its Usage. J. Chongqing Univ. of Technol. (Nat. Sci.) 2013, 27, 131-134.

19. Zhao, H.; Wang, Z.; Xie, K.; Li, W. Comparative Study on Reliability Assessment Methods for Medium Voltage Distribution Network. Power Syst. Technol. 2013, 37, 3295-3302.

20. Qin, L.; Sun, M.; Li, S.; Zhu, X.; Wang, G. Multi-objective planning of wind and photovoltaic generation based on hybrid intelligent algorithm considering load profile and generation profile. Renew. Energy Resour. 2015, 33, 843-850.

21. Li, M. The Comprehensive Evaluation of AC and DC Distribution System Based on Improved Analytic Hierarchy Process. Ph.D. Thesis, North China Electric Power University, Beijing, China, 2015.

22. Qiu, Y.; Li, J.; Deng, X.; Deng, G.; Zhou, H. Comprehensive Evaluation of Power Quality Based on Improved TOPSIS-RSR Method. High Volt. Appar. 2018, 54, 44-50.

23. Zheng, Z.-H.; Ai, Q.; Gu, C.-H.; Jiang, C.-W. Multi-objective Allocation of Distributed Generation Considering Environmental Factor. Proc. CSEE 2009, 29, 23-28.

24. Li, R.; Shen, X.; Zhong, C.; Yang, T. Multi-Objective Planning of Distributed Generation Considering Environmental Benefit. Power Syst. Technol. 2014, 38, 1471-1478.

25. Zeng, X.; Yang, Q.; Ren, J. Line Vulnerability Assessment on Power Grid Based on Combination Weighing Method. Proc. CSU EPSA. 2016, 28, 44-48. [CrossRef]

26. Jiao, F.; Xiao, J.; Wang, R.; Ye, J.; Jian, G.; Zhan, K. Application of Comprehensive Evaluation Model to Smart Grid Based on Combination Weight. Proc. CSU EPSA. 2017, 29, 143-150. 
27. Li, J.; Zhang, H. Combination Weighting Method Application in Social Evaluation of Offshore Wind Power Projects. J. Eng. Manag. 2012, 26, 18-22.

28. Luo, Y.; Zhou, C.; Liu, X. Application of the Multi-level Grey Relational Analysis Method in Operation Assessment of Thermal Power Units. Proc. CSEE 2012, 32, 97-103.

29. Yang, Y.; Wu, D.; Wang, N. Comprehensive evaluation for large scale coal-fired power units based on combined weight and TOPSIS method. Therm. Power Gener. 2016, 45, 10-15.

30. Zhao, S.; Tang, S. Comprehensive evaluation of transmission network planning scheme based on improved analytic hierarchy process, CRITIC method and TOPSIS. Electr. Power Autom. Equip. 2019, 1-7. [CrossRef]

31. Luo, Y.; Li, Y. Comprehensive Decision-Making of Transmission Network Planning Based on Entropy Weight and Grey Relational. Power Syst. Technol. 2013, 37, 77-81.

(C) 2019 by the authors. Licensee MDPI, Basel, Switzerland. This article is an open access article distributed under the terms and conditions of the Creative Commons Attribution (CC BY) license (http://creativecommons.org/licenses/by/4.0/). 